\section{Academic Freedom}

THERE seems to me to be much confusion in recent discussions of this matter. Consider the following propositions :-

In respect of any opinion $X$ and its contradictory $Y$, there are :

Some people (Class $A$ ) who should be allowed to express either $X$ or $Y$;

Some people (Class $B$ ) who must hold $X$ if they are to perform their duties adequately (for example, cabinet ministers and surveyors, who should not be flat earthers).

Some people (Class $C$ ) whose duties involve impartiality so intimately that they should not express publicly either $X$ or $Y$ (for example, judges).

In Great Britain these propositions are accepted so widely that a dispute scarcely ever arises from their denial. It arises either because men do not act in accordance with their professions, or because there are differences concerning the bounds of $A, B$, $C$. The question of academic freedom here is how academic persons are to be classified; the extreme view is that, in respect of all opinions, they ought to be placed in $A$.

In dictatorial countries the propositions are definitely denied, and classes $A, B, C$ are not recognised. The only classes recognised are those $(D)$ of persons who must publicly advocate $X$, and those $(E)$ who may (not must) remain silent. Our conception of academic freedom, since it involves the recognition of $A, B, C$, does not arise. To urge a dictatorial government to create a class $A$ in favour of teachers (and not, say, bus-drivers) is to invite them to add imbecility to intolerance; it is simply ridiculous. If we are to persuade them to abandon intolerance, the less we say about academic freedom the better.

Actually opinions in Great Britain are not uniform about the classification of academic persons. Some people (of whom I am one) consider that, in respect of some opinions, they should be $C$, not $A$. Where there is a consensus of instructed opinion, and where they hold their post owing to the existence of that consensus, professors should not express personal opinions, except on the condition that they always and deliberately make the distinction clear. For example, I should like to see writers on popular science forbidden (by public opinion, not of course by law) to propagate insidiously opinions that they know to be rejected by persons equally qualified in science. For this reason, too-though I am as antidictatorial as any person can be-I think we should settle our own views on academic freedom before we try to enforce them on others.

155 Hagden Lane,

Watford, Herts.

Feb. 9.

X-Ray Analysis of the Orthorhombic Crystalline Modification of I: $2: 5: 6$-Dibenzanthracene

Is a recent paper ${ }^{1}$, Krishnan and Banerjee have described a new crystalline modification of the carcinogenic hydrocarbon 1:2:5:6-dibenzanthracene. By means of optical measurements, they show that the crystals are different from the monoclinic variety already known and examined by means of X-rays ${ }^{2}$. In this laboratory similar crystals have been obtained in the way described by the above authors from a solution in ethyl acetate, and they have now been examined by X-rays. The crystals are exceptionally well developed, having more or less uniform dimensions in all directions and show brilliant faces.

The X-ray analysis confirms the conclusion of Krishnan and Banerjee that the crystals are orthorhombic bipyramidal, and the following dimensions have been found for the unit cell :

$$
a=8 \cdot 22 \text { A. ; } b=11 \cdot 39 \text { A. ; } c=15 \cdot 14 \text { A. }
$$

Assuming four molecules of $\mathrm{C}_{22} \mathrm{H}_{14}$ per unit cell, these values give a crystal density of $1 \cdot 295$, which is in good agreement with the observed value ${ }^{1}, 1 \cdot 282$. The axes have been named differently from those of Krishnan and Banerjee, so that the optical vibration direction $\gamma$ is along $c$, and $\alpha$ along $a$, this arrangement being more convenient for comparison with other similar structures, and in this case the axes are crystallographically indistinguishable. The $a, b$ and $c$ of the above authors have been renamed $b, c$ and $a$ respectively. The axial ratios are, $b: c: a$ $0 \cdot 752: 1: 0 \cdot 543$ compared with $0 \cdot 755: 1: 0 \cdot 546$ given by Krishnan and Banerjee.

The observed halvings are $(0 k l)$ when $l$ is odd, $(h 0 l)$ when $h$ is odd and $(h k 0)$ when $k$ is odd. The space group is therefore $Q_{h}^{15}-P c a b$. It is interesting to note that in this space group with four molecules per unit cell, the molecules can possess only a centre of symmetry, whereas in the case of the monoclinic variety $^{2}$ there are two space groups available, in one of which no molecular symmetry is necessary and in the other the molecule can possess either a centre or a plane of symmetry.

As regards the molecular orientation which has been deduced by Krishnan and Banerje日 ${ }^{3}$ from measurements of magnetic and optical anisotropy, one can only say at this stage of the analysis that the intensity of reflections observed seems to support their conclusions that the length of the molecule is perpendicular to the (001) plane and its width inclined to the $b$ axis.

I am indebted to Prof. J. W. Cook for supplying a pure specimen of the substance.

Research Institute,

JoHN IBaLL.

The Cancer Hospital (Free), London, S.W.3. Jan. 8.

$1 Z$. Krist., 91, 170 (1935).

2 Iball and Robertson, NATURE, 132, 750 (1933).

s Z. Krist., 91, 173 (1935).

Relation between the Toxicities and the Boiling Points of Related Substances

IN attempts to relate the toxicity of physiologically active substances to their chemical constitution or physical properties, it has been the custom to use as comparative indexes of toxicity the numerical values of the molar doses or concentrations producing equal physiological effects. It is well known that the degree of physiological response to different doses or concentrations of the same substance, in many cases at least, varies directly as the logarithm of the dose or concentration applied. Hence, when the toxicities of different substances are being compared, it would appear probable that the use of the logarithms of equi-effective molar doses is more likely to yield quantitative relationships between toxicity and, say, physical properties than the simple numerical values. 